\title{
OSCILLATION OF NONLINEAR MATRIX DIFFERENTIAL EQUATIONS OF SECOND ORDER
}

\author{
E. C. TOMASTIK
}

We shall be concerned in this paper with the oscillation of the matrix differential equation

$$
\left[R(t) U^{\prime}\right]^{\prime}+F\left(t, U, U^{\prime}\right) U=0,
$$

where $U=\left(u_{i j}\right), F=\left(f_{i j}\right)$ and $R$ are $n \times n$ matrices. By $F=F\left(t, U, U^{\prime}\right)$ is meant $f_{i j}=f_{i j}\left(t, u_{11}, \cdots, u_{n n}, u_{11}^{\prime}, \cdots, u_{n n}^{\prime}\right)$. The functions $f_{i j}$ are assumed to be continuous for $t$ on $[a, \infty), a \geqq 0$, and for all values of the remaining variables. The matrix $F\left(t, U, U^{\prime}\right)$ is symmetric and positive definite for every $t$ on $[a, \infty)$ and every matrix $U$ with det $U \neq 0$, while the matrix $R(t)$ is continuous, symmetric and positive definite for every $t$ on $[a, \infty)$.

Equation (1) is equivalent to a system of $n^{2}$ second order equations or to $2 n^{2}$ first order differential equations and so any existence and uniqueness theorem for such systems will apply to (1). We merely assume here that we do have existence and uniqueness for the system tive (1) together with the boundary condition

$$
U(b)=\Lambda, \quad U^{\prime}(b)=B,
$$

when $A$ and $B$ are arbitrary matrices and $b \geqq a$. In view of recent results in the scalar case of (1) (cf. [2]), we will also assume that all solutions of the system (1) and (2) can be continued to $t=\infty$.

Before we are able to state what is meant by (1) being oscillatory, a simple fact about the solutions of (1) is needed. If $U=U(t)$ is any solution matrix of (1), then

$$
U^{*}(t) R(t) U^{\prime}(t)-U^{* \prime}(t) R(t) U(t) \equiv C,
$$

where $C$ is a constant matrix and "*" designates the transpose. This is easily seen by differentiating both sides of (3) and using (1) and the symmetry of $F\left(t, U, U^{\prime}\right)$ and $R(t)$. If the constant matrix $C$ is the zero matrix, i.e.,

$$
U^{*}(t) R(t) U^{\prime}(t) \equiv U^{* \prime}(t) R(t) U(t),
$$

then we shall say (as in the linear case) that $U(t)$ is prepared.

We are now able to state what is meant by (1) being oscillatory. The nonlinear matrix equation (1) will be termed oscillatory on

Received by the editors August 19, 1967. 
$[a, \infty)$ if given any prepared matrix solution $U(t)$ of $(1)$, the determinant of $U(t)$, det $U(t)$, has arbitrarily large zeros. More precisely, given any $b \geqq a$, det $U(t)$ vanishes on $(b, \infty)$.

This definition is the same as the definition of oscillatory in the case that (1) is linear (cf. [3]). In the scalar case, $n=1$, this definition guarantees that all solutions of (1) have arbitrarily large zeros.

All of our theorems presented here will depend on certain properties of nonoscillatory solutions of (1). These results are presented in the following lemma.

Leмma. If $U(t)$ is a prepared matrix solution of (1) such that $\operatorname{det} U(t) \neq 0$ on some interval $(b, \infty)$, then $\operatorname{det} U^{\prime}(t) \neq 0$ on some interval $[c, \infty), c>b$. Furthermore, if $S(t)=R(t) U^{\prime}(t) U^{-1}(t)$, then the following two equations are satisfied for all $t \geqq c$ :

$$
\begin{aligned}
S(t)=S(c) & -\int_{c}^{t} F\left[x, U(x), U^{\prime}(x)\right] d x-\int_{c}^{t} S(x) R^{-1}(x) S(x) d x \\
S^{-1}(t)= & S^{-1}(c)+\int_{c}^{t} S^{-1}(x) F\left[x, U(x), U^{\prime}(x)\right] S^{-1}(x) d x \\
& +\int_{c}^{t} R^{-1}(x) d x
\end{aligned}
$$

Since det $U(t) \neq 0$ on $(b, \infty), S(t)$ is well defined on $(b, \infty)$. It readily follows from the preparedness of $U(t)$ and from (4) that $S(t)$ is also symmetric. Furthermore, it is easy to see from (1) that

$$
S^{\prime}(t)=-F\left[t, U(t), U^{\prime}(t)\right]-S(t) R^{-1}(t) S(t) .
$$

The right-hand side of (7) is negative definite since $F$ is positive definite and $S R^{-1} S=S^{*} R^{-1} S$ is positive semidefinite. Each characteristic root of $S(t)$, then, is strictly decreasing and det $S(t)$ can vanish at most $n$ times. There then exists $c>b$ such that $S(t)$, and thus $U^{\prime}(t)$, is not singular on $[c, \infty)$. Equations (5) and (6) then follow from either (1) or (7). The Lemma is now established.

For a symmetric matrix $A$, let the characteristic roots of $A$ be ordered in the following way:

$$
\lambda_{1}[A] \geqq \lambda_{2}[A] \geqq \cdots \geqq \lambda_{n}[A] .
$$

Before stating the next theorem, note that if $A(t)$ is positive semidefinite, then $\lambda_{1}\left[\int_{a}^{\infty} A(s) d s\right]=\infty$ if and only if $\int_{a}^{\infty} \lambda_{1}[A(s)] d s=\infty$. This follows since $A(t)$ positive definite implies that $\int_{a}^{t} A(s) d s$ is positive definite (for $t>a$ ), and 


$$
\begin{aligned}
\sum_{i=1}^{n} \int_{a}^{t} \lambda_{i}[A(s)] d s & =\int_{a}^{t} \operatorname{tr} A(s) d s=\operatorname{tr} \int_{a}^{t} A(s) d s \\
& =\sum_{i=1}^{n} \lambda_{i}\left[\int_{a}^{t} A(s) d s\right],
\end{aligned}
$$

where $\operatorname{tr} B$ is the trace of $B$.

THEOREM 1. Let $R(t) \equiv I$, the identity matrix. If

$$
\lambda_{1}\left[\int_{a}^{\infty} F\left[t, A(t), A^{\prime}(t)\right] d t\right]=\infty,
$$

for every differentiable matrix $A(t)$ such that $\lambda_{n}\left[A^{*}(t) A(t)\right] \geqq \epsilon>0$ for large $t$, then (1) is oscillatory.

Assume, contrary to the theorem, that (1) is not oscillatory, i.e., there exists a $b \geqq a$ and a prepared matrix solution $U(t)$ of (1) such that det $U(t) \neq 0$ on $(b, \infty)$. Using the Lemma, equations (5) and (6) are then satisfied. Now, using the Courant-Hilbert min-max theorem, we see from (6) that since the first integral in (6) is positive definite, all characteristic roots $S^{-1}(t)$ have limits equal to $+\infty$; thus, $S(t)$ is positive definite for large $t$ and

$$
\lim S(t)=0 .
$$

Since det $U(t) \neq 0$ on $[c, \infty)$, we can consider $U(t)$ to be a solution matrix of the linear equation

$$
U^{\prime}=S(t) U
$$

We then obtain

$$
\left[U^{*}(t) U(t)\right]^{\prime}=2 U^{*}(t) S(t) U(t),
$$

which is positive definite for large $t$ since $S(t)$ is positive definite for large $t$ and det $U(t) \neq 0$. Thus each (positive) characteristic root of $U^{*}(t) U(t)$ is strictly increasing and $\lambda_{n}\left[U^{*}(t) U(t)\right] \geqq \epsilon>0$ for some $\epsilon$ and for large $t$. By hypothesis, we then conclude that

$$
\lambda_{1}\left[\int_{a}^{\infty} F\left[t, U(t), U^{\prime}(t)\right] d t\right]=\infty .
$$

Using this fact in (5) and the Courant-Hilbert min-max theorem, immediately yields one characteristic root of $S(t)$ unbounded, contradicting (8). From this contradiction, we infer the truth of the theorem. 
Corollary. Suppose $F\left(t, U, U^{\prime}\right)=U^{* k} Q(t) U^{k}$ where $Q(t)$ is positive definite on $[a, \infty)$ and $k$ is some nonnegative integer. Then if the matrix $\int_{a}^{t} Q(x) d x$ is unbounded, (1) is oscillatory.

Assume $A(t)$ is a differentiable matrix such that $\lambda_{n}\left[A^{*}(t) A(t)\right]$ $\geqq \epsilon>0$ for large $t$. This implies immediately that $\lambda_{1}\left[A^{* k}(t) A^{k}(t)\right] \geqq \epsilon^{k}$ for large $t$. Since $Q(t)$ is positive definite, it follows readily from the Courant-Hilbert min-max theorem that

$$
\lambda_{1}\left[A^{* k}(t) Q(t) A^{k}(t)\right] \geqq \epsilon^{k} \lambda_{1}[Q(t)]
$$

for large $t$. Since $Q(t)$ is positive definite and $\int_{a}^{t} Q(x) d x$ is unbounded, $\lambda_{1}\left[\int_{a}^{\infty} Q(x) d x\right]=\infty$. Now using this fact and the last inequality above and recalling the comments immediately preceding the statement of Theorem 1, we conclude that $\lambda_{1}\left[\int_{a}^{\infty} A^{* k}(t) Q(t) A^{k}(t) d t\right]=\infty$. Thus the hypothesis of Theorem 1 is satisfied and (1) is oscillatory.

In line with other results (cf. [3]), one would expect the same oscillation theorem as Theorem 1 would also hold in the case that all characteristic roots of $\int_{a}^{t} R^{-1}(x) d x$ are unbounded. The following theorem is a result in this direction.

THEOREM 2. If $F$ satisfies the same hypothesis as in Theorem 1 and if $R(t)=r(t) I$, where $r(t)$ is a positive scalar function and $\int_{a}^{\infty} r^{-1}(x) d x=\infty$, then (1) is oscillatory.

The proof is the same as for Theorem 1, except that equation (10) now becomes

$$
\left[U^{*}(t) U(t)\right]^{\prime}=U^{*}\left[S R^{-1}+R^{-1} S\right] U .
$$

In the case that $R(t)=r(t) I, r(t)>0, S R^{-1}+R^{-1} S$ is again positive definite and the proof proceeds as before.

One should notice that with $S$ and $R$ positive definite, the matrix $S R^{-1}+R^{-1} S$ need not be positive definite even if $R$ is diagonal.

We continue studying equation (1) with the following theorem.

ThEOREM 3. Suppose that for every differentiable matrix $A(t)$ with $\operatorname{det} A(t) \neq 0$ for large $t$, we have

and

$$
\lambda_{i}\left[\int_{a}^{\infty} F\left[t, A(t), A^{\prime}(t)\right] d t\right]=\infty, \quad i=1,2, \cdots, p,
$$

$$
\lambda_{i}\left[\int_{a}^{\infty} R^{-1}(t) d t\right]=\infty, \quad i=1,2, \cdots, r .
$$

Then, if $r+p>n$, (1) is oscillatory. 
Again, assuming contrary to the theorem that (1) is not oscillatory, there exists a $b \geqq a$ and a prepared matrix solution $U(t)$ of (1) such that det $U(t) \neq 0$ on $(b, \infty)$. The Lemma now applies and equations $(5)$ and (6) are satisified.

Using the Courant-Hilbert min-max theorem again, we see from (5) using (11) that $S(t)$ has $p$ characteristic roots whose limits are $-\infty$. From (6) using (12), we see that $S^{-1}(t)$ has $r$ characteristic roots whose limits are $+\infty$. But this contradicts the fact that $r+p>n$.

As pointed out in [3], Theorem 3 is not true even in the linear case if $r+p=n$.

\section{REFERENCES}

1. G. J. Etgen, Oscillatory properties of certain nonlinear matrix differential systems of second order, Trans. Amer. Math. Soc. 122 (1966), 289-310.

2. Stuart $P$. Hastings, Boundary value problems in one differential equation with a discontinuity, J. Differential Equations 1 (1965), 346-369.

3. E. C. Tomastik, Singular quadratic functionals of $n$ dependent variables, Trans Amer. Math. Soc. 124 (1966), 60-76.

4. - Oscillation of a nonlinear second order differential equation, SIAM J. Appl. Math. 5 (1967), 1275-1277.

The University of Connecticut 